\title{
Vehicular Ad Hoc Network Mobility Model
}

\author{
Budi Rahmadya \\ Computer System Departement, Faculty of Information Technology, Andalas University, Padang, Indonesia \\ E-mail:budi22_ok@yahoo.com
}

\begin{abstract}
Indonesia is one of developing countries with high land traffic density. This traffic density could cause traffic jam, traffic accidents and other disturbances. This research had developed a simulator that could calculate the traffic density of roads in urban areas. With the use of this simulator, the researcher could calculate the time needed if the source node transports the message to the destination node by using the ad hoc network communication facility. In this research, every vehicle utilizes multi-hop communication in a communication network. The vehicle sends the message through flooding message and passes on the received message to other vehicles. Based on the simulation done on map size $10 \mathrm{~km} \times 10 \mathrm{~km}$ with a total of 20 vehicles on the road, it was calculated that the simulator could transmit the message to its destination on the 106th second from node 3 and with the total of 200 vehicles on the road, the simulator could transmit the message to its destination on the 22nd second from node 5.
\end{abstract}

Keywords - Wireless Ad Hoc Network communication, multi-hop communication, flooding message

\section{INTRODUCTION}

Wireless ad hoc network communication technology has recently become a popular research topic and a worldwide interest in the field of data and network communication. Vehicular Ad Hoc Network Communication (VANETs) is a technology that uses wireless network communication with the concept of multi-hop wireless communication. This technology utilizes the vehicles as a router in communication network. The vehicle delivers information to other vehicles in a network communication. Information delivered could contain road accident, traffic density and many others.

The characteristic of the VANETs itself is the difficulty in predicting the destination of each vehicle on the road, which is mainly caused by the density of vehicles mobility. In the developing country like Indonesia, high road density could be found in major cities like Jakarta, Bandung and Bogor, etc. This situation inevitably will become one of the factors that will hinder the progress of a country. For the time being, the problem on those major cities is handled with CCTV media which is controlled by National Traffic Management Center (NTMC) POLRI [1].

This research has discussed a simulation model by observing the vehicles mobility on the roads designed for urban area. The simulation was conducted using SUMO vehicular traffic simulator and had calculated the time needed by the destination node in receiving data package from the source node. This simulator is expected to give a description to the police or the road and transportation management bureau or the VANETs researchers about traffic condition on the road. The simulation was conducted using varying number of vehicles.

The rest of the paper is organized as follows. Section II discusses related work. Section III we discusses proposed method. Simulation and result is given in section IV. Finally in Section $\mathrm{V}$, we explain some conclusions and future work.

\section{RELATED WORK}

Several methods have been proposed related to ad hoc network simulation. The authors [2] discussed the existing VANETs simulators. This discussion could provide an input for the researchers in VANETs in the future uses of the simulator. The authors compared some simulators based on their software characteristics, the Graphics User Interface (GUI), popularity, ease of use, output visualization abilities, simulation accuracy, etc. The authors [3] designed a VANETs simulator that is different from the present simulator. It takes into consideration the complex condition of the traffic with the number of vehicle that could amount to thousands in a city. The authors [4] discussed about SUMO-Simulation of Urban Mobility. This simulation was specifically designed for urban area traffic condition. The authors described that this simulator could provide description about traffic condition, several research topics csuch as route selection and/or vehicle communication simulation. 
Mobility model for VANETs was described by Khaimar, V.D, et al [5]. The authors used random way-point model in the simulation. The authors compared the tool on the SUMO with the MOVE simulator. The output of MOVE simulation could and would be implemented on Network Simulator 2 and Qualnet Simulator. Arbabi H, et al. [6] presented a simulator model that used NS-2. In the simulation conducted in highway class, the authors calculated the existence of vehicle mobility in the communication network. Mahajan, A. et al [7] have introduced a new mobility model in VANETs. Based on the result of conducted simulation, it was found that it had a higher accuracy level in sending data package. Lan, K.C et al [8] have introduced a realistic mobility model in VANETs simulation. He utilized MOVE tools and concentrated in analysing traffic lights, driver route choice and car overtaking behaviour. The result of this analysis could serve as a reference in designing protocol for high mobility network.

\section{A. SUMO Vehicular Traffic Simulator}

SUMO is a traffic simulator developed by German Aerospace Centre, Institute of Transportation System [9]. This simulator could operate on major traffics. Generally, the Graphic User Interface of the SUMO-Simulator could be seen on Figure 1 below:

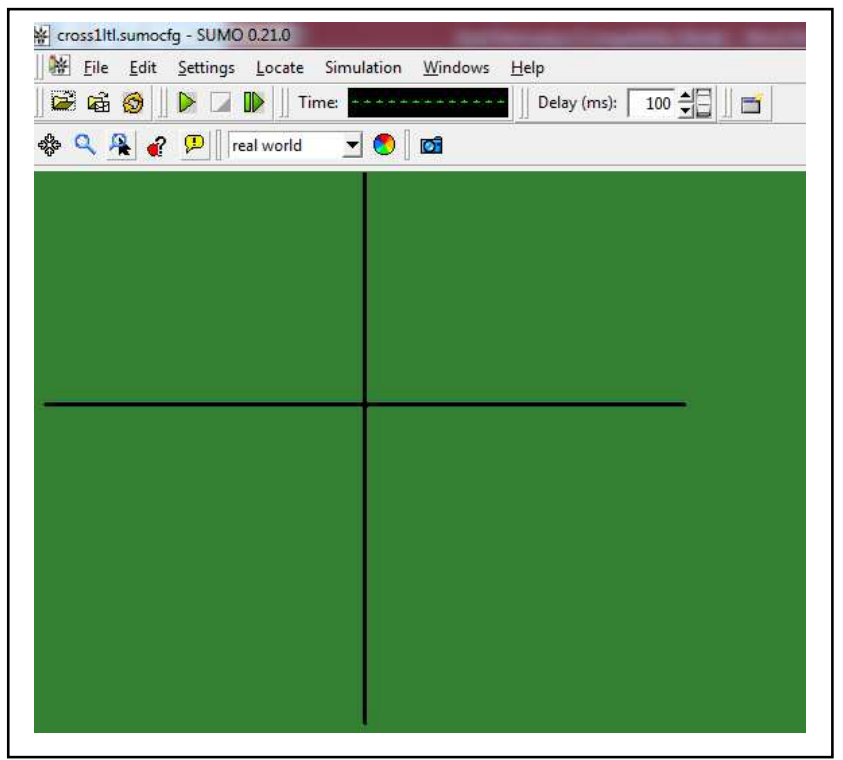

Fig. 1 GUI SUMO-Simulator

On the GUI above we could see that this simulator provides options for Delay setting which functions to manage the speed of played simulation. To create a better simulation situation, there was also an option for the simulation model, such as standard, faster standard and real world. Below are several road traffic models that are provided by SUMO-Simulator:

\section{1) Busses Model}

This traffic model has a form like a rectangular figure which consists of two way traffic channels, several intersection and bus stops. Figure 2 below is the busses model.

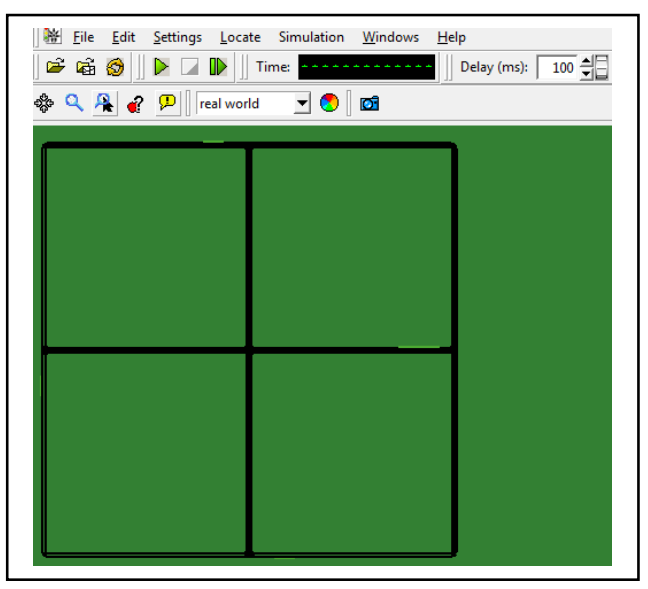

Fig. 2 Busses Model

\section{2) Cross Full Model}

The Cross Full Model presents four intersection models, in which each channel consists of four traffic channels. Figure 3 below is the Cross Full Model.

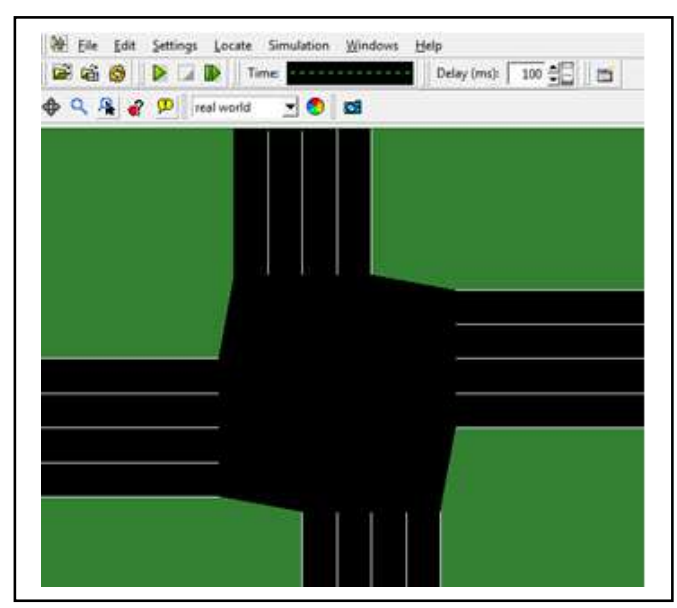

Fig.3 Cross Full Model

\section{3) Box Model}

The Box Model has a form like a box or a square. This model consists of 4 way traffic channels. Figure 4 below is an illustration of the box model.

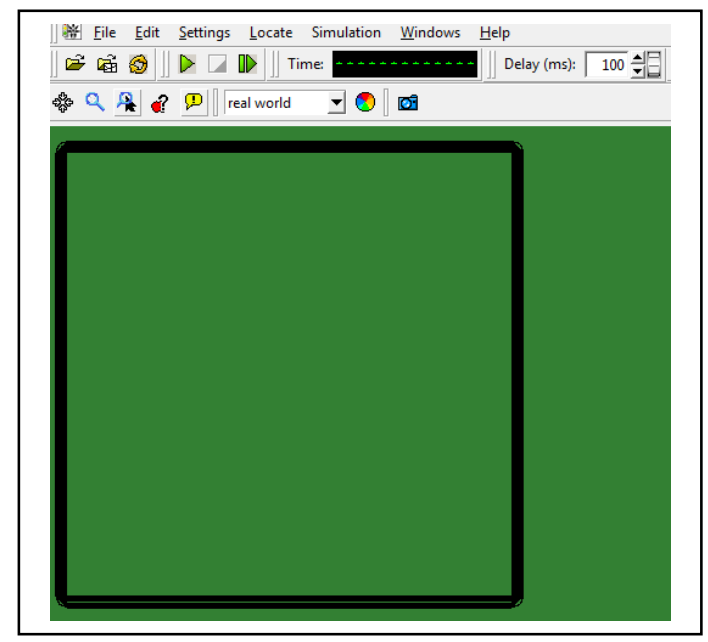

Fig.4 Box Model 


\section{B. Importing Map to SUMO}

One of the important features of SUMO-Simulator is that it could be integrated to a map of an area. This map is used to condition the traffic simulation of an area. Map importing to SUMO could be conducted through OpenStreeetMap facility [10]. Figure 5 below is the selection of an area in Jakarta using OpenStreetMap facility.

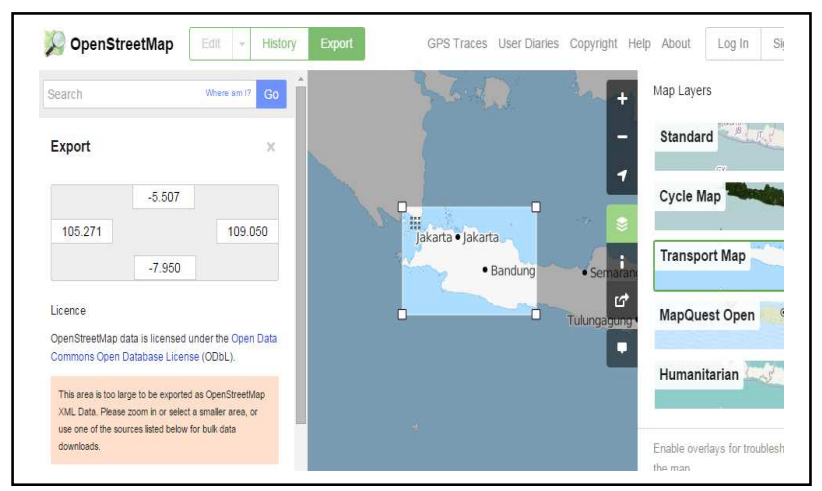

Fig. 5 OpenStreetMap

\section{PROPOSED METHOD}

In this proposed method, the authors will conduct a vehicles simulation on the street road using SUMO vehicular traffic simulator. Communication among the vehicles was done through flooding technique. In this research, we are assuming a city-scale deployment, and we are going to use a map of $10 \mathrm{~km} \times 10 \mathrm{~km}$ size.

\section{A. Information Communication Exchange}

This part would describe the condition of the communication network of existing vehicles on the street road. Each vehicle was equipped with communication device conforming to IEEE 802.11. The information exchange between vehicles was occurred in 100 meters radius. The source node sent the message to the neighbour vehicles; every message received by neighbour vehicle would be stored and forwarded to another vehicles. Figure 6 below describes the communication process occurred.

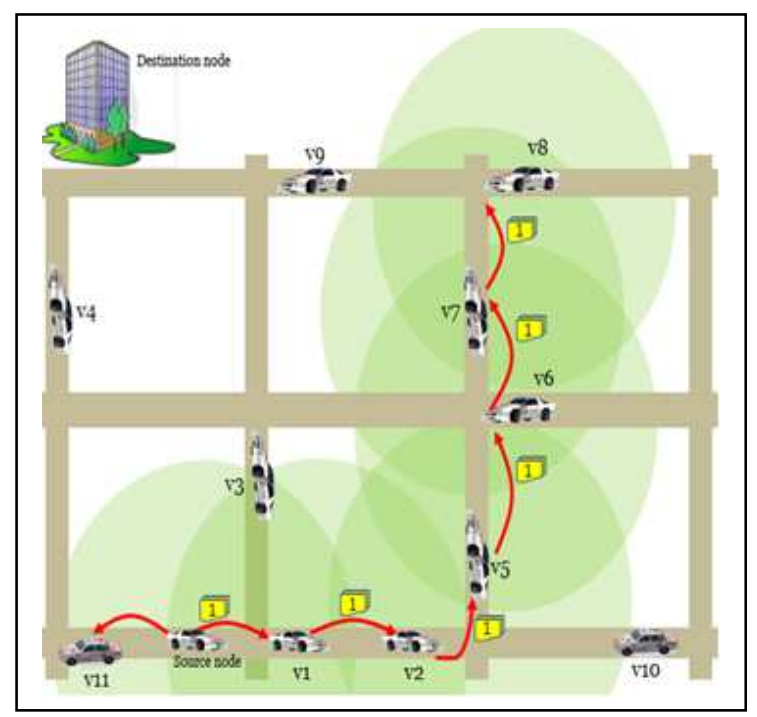

Fig. 6. Information Communication Exchange

\section{SIMULATION AND RESULT}

On the illustration below it can be seen that the simulation was conducted using Manhattan map that was operated by using java programming. The other two graphics below illustrates the network condition on the last 6 seconds with the number of connected vehicle on each second.

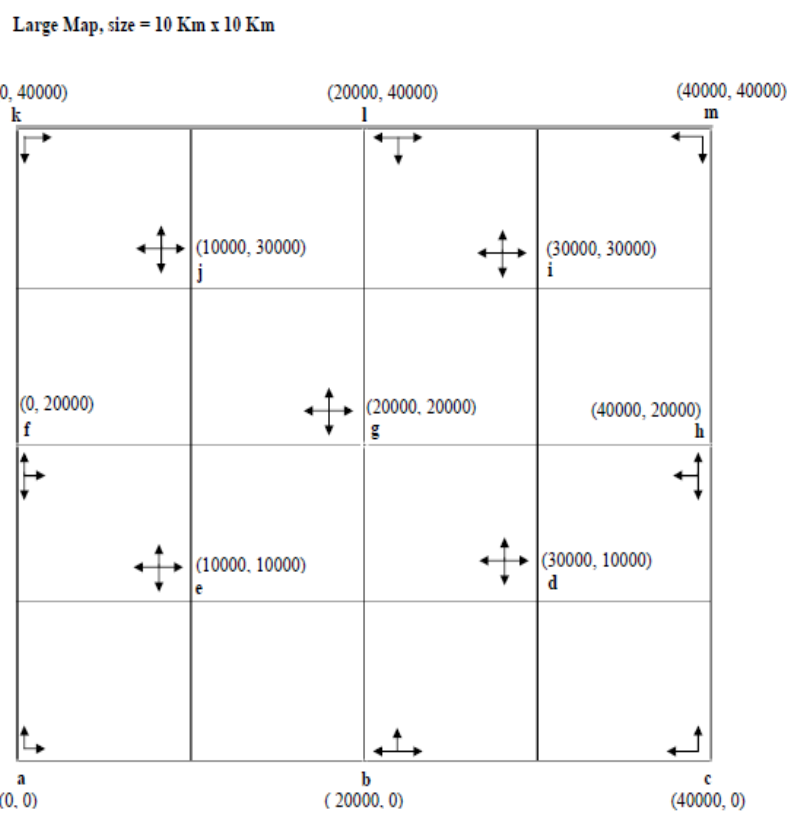

Fig. 7. Manhattan Map Model

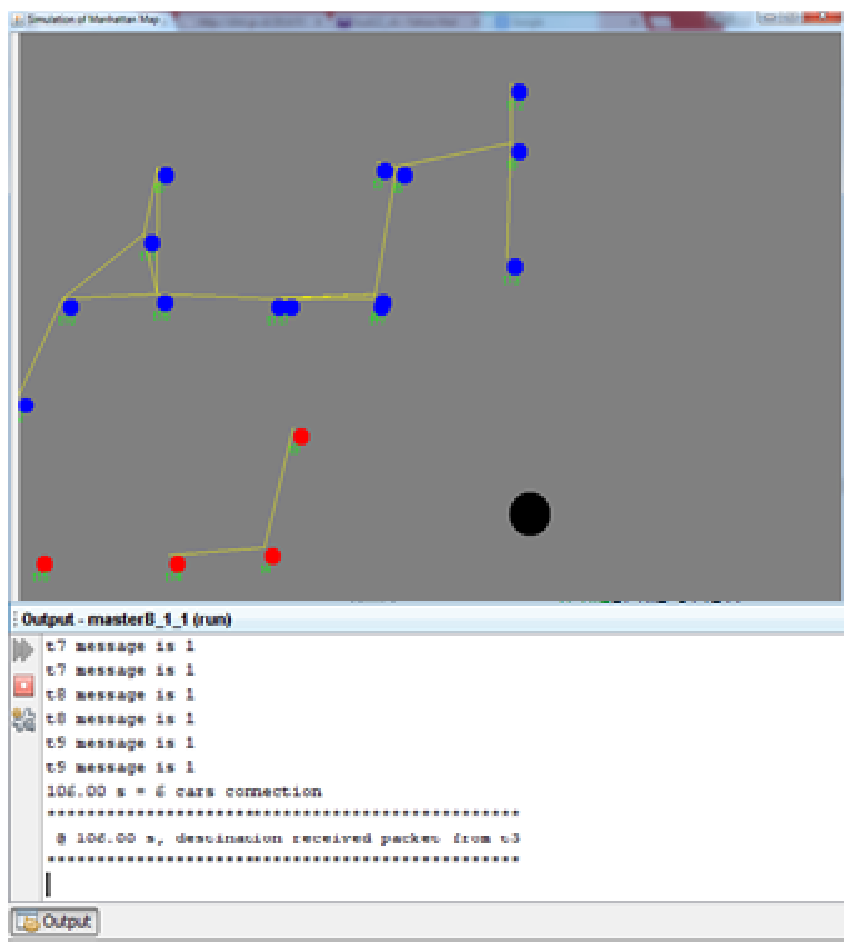

Fig.8. Simulation Process 


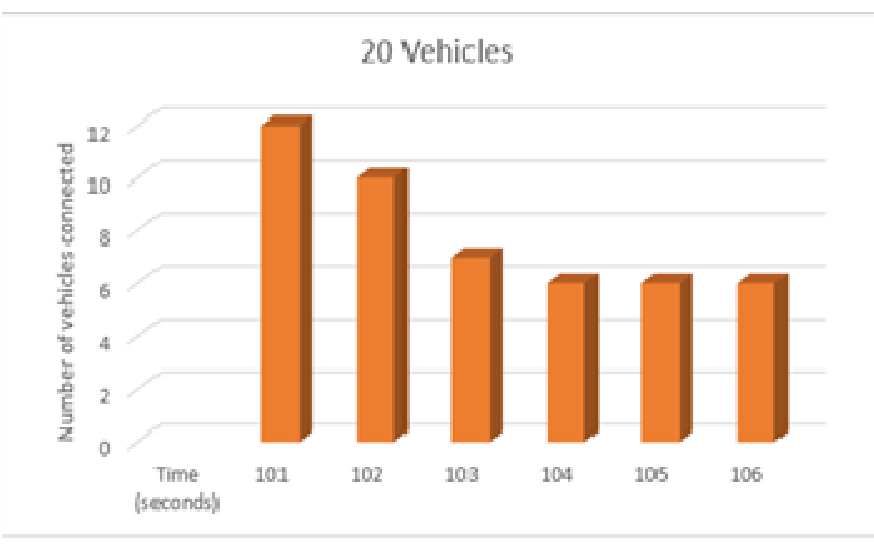

Fig. 9 Node Connection Graphic of the 20 Vehicles on the Last 6 Seconds

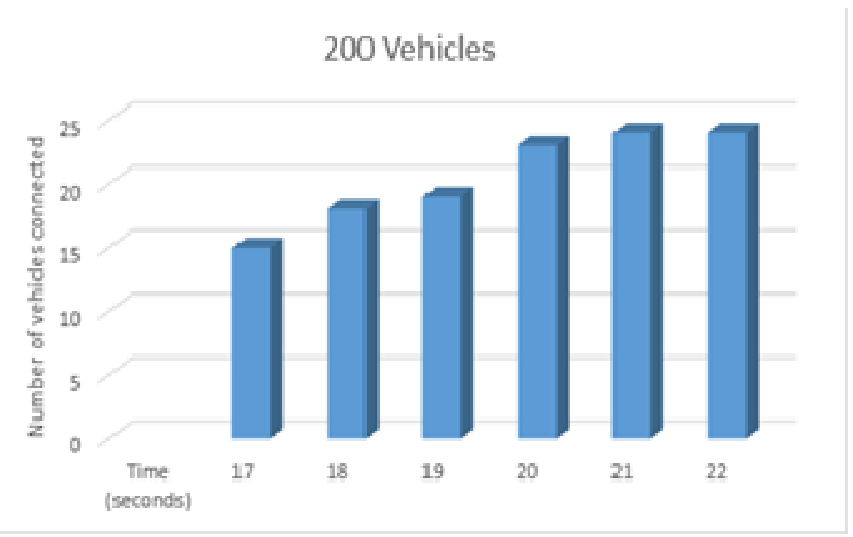

Fig. 10. Node Connection Graphic of the 200 Vehicles on the Last 6 Seconds

\section{CONCLUSIONS AND FUTURE WORK}

Simulation was conducted through comparing the different number of vehicles in every second. The simulation was done for 1 hour for each simulation. With the number of vehicle of 20 vehicles, it could deliver message to the destination on the 106th second, and its destination node received data from node 3 . With the number of vehicle of 200 vehicles, it could deliver message to its destination on the 22 nd second, destination node received data from node 5 .
In the next development planning, the simulation will implement real map such Jakarta area, Bandung area, Bogor area, etc. The implementation also includes the use of routing protocol in delivering the message from source node to destination note. The selection of proper routing protocol that perfectly fits the existing traffic condition is expected to save the available bandwidth of ad-hoc network. To assure that the data package safely reaches its destination, it would be necessary to implement the concept of Delay Tolerant Network/DTN on the next simulation [11].

\section{REFERENCES}

[1] http://www.korlantas-irsms.info/graph/accidentData

[2] http://ntmc-korlantaspolri.blogspot.com

[3] Martinez, F. J. Toh, C. K. Cano, J. C. Calafate, C. T. and Manzoni, P. "A survey and comparative study of simulators for vehicular ad hoc networks (VANETs)" WIRELESS COMMUNICATIONS AND MOBILE COMPUTING Wirel. Commun. Mob. Comput. (2009) Published online in Wiley InterScience (www.interscience.wiley.com) DOI: $10.1002 / \mathrm{wcm} .859$

[4] Gorgorin, C. Gradinescu, V. Diaconescu, R. Cristea, V and Iftode, L. "SUMO- Simulation of Urban Mobility, "An Integrated Vehicular and Network Simulator for Vehicular Ad-Hoc Networks"

[5] Behrisch, M. Bieker, L. Erdmann, J. and Krajzewicz, D. Institute of Transportation Systems "SUMO- Simulation of Urban Mobility," SIMUL 2011 : The Third International Conference on Advances in System Simulation 2011

[6] Khairnar, V.D and Pradhan, Dr. S. N. "Mobility Models for Vehicular Ad-hoc Network Simulation," International Journal of Computer Applications (0975 - 8887) Volume 11- No.4, December 2010

[7] Arbabi, H and Weigle, M.C "Highway Mobility and Vehicular AdHoc Networks in NS-3," Proceedings of the 2010 Winter Simulation Conference B. Johansson, S. Jain, J. Montoya-Torres, J. Hugan, and E. Yücesan, eds

[8] Mahajan, A. Potnis, N. Gapolan, K. and Wang, An-I A, "Urban Mobility Models for VANETs,"

[9] Lan, K. C. and Chou, C. M. "Realistic Mobility Models for Vehicular Ad hoc Network (VANET) Simulations," ITS Telecommunications, 2008. ITST 2008. 8th International Conference on , vol., no., pp.362,366, 24-24 Oct. 2008

[10] SUMO Simulation of Urban MObility: SUMO HOMEPAGE,http://sumo.sourceforge.net/

[11] http://www.openstreetmap.org/\#map=9/-7.2181/107.3447\&layers=T

[12] Budi, R. Sun, W. Shibata, N. and Ito M. "A DTN Based Data Dissemination Method Utilizing Platoon in Urban Area", IPSJ SigITS 53, Technical Report, Vol. 2013-ITS-53, No.5, pp.1-6, 2013.6.7 